\title{
Nucleon quark distributions in a covariant quark-diquark model
}

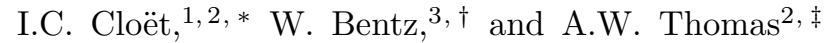 \\ ${ }^{1}$ Special Research Centre for the Subatomic Structure of Matter and \\ Department of Physics and Mathematical Physics, University of Adelaide, SA 5005, Australia \\ ${ }^{2}$ Jefferson Lab, 12000 Jefferson Avenue, Newport News, VA 23606, U.S.A. \\ ${ }^{3}$ Department of Physics, School of Science, Tokai University, Hiratsuka-shi, Kanagawa 259-1292, Japan
}

\begin{abstract}
Spin-dependent and spin-independent quark light-cone momentum distributions and structure functions are calculated for the nucleon. We utilize a modified Nambu-Jona-Lasinio model in which confinement is simulated by eliminating unphysical thresholds for nucleon decay into quarks. The nucleon bound state is obtained by solving the Faddeev equation in the quark-diquark approximation, where both scalar and axial-vector diquark channels are included. We find excellent agreement between our model results and empirical data.
\end{abstract}

PACS numbers: 13.60.Hb,12.38.Lg,11.80.Jy,12.39.Fe,12.39.Ki

\section{INTRODUCTION}

The discovery in the late 1980's by the European Muon Collaboration (EMC) that the fraction of the spin of the proton carried by the quarks is unexpectedly small 1], caused much excitement in the nuclear and particle physics communities. The "proton spin crisis" prompted many new experiments, leading to major new insights into the spin structure of the proton. However, a thorough theoretical understanding of the non-perturbative parton distributions still remains an exciting challenge.

In this paper we calculate the spin-independent and spin-dependent quark distributions in the framework developed by Bentz, Thomas and collaborators, in which proper-time regularization is applied to the Nambu-Jona-Lasinio (NJL) model 2] in order to simulate the effects of confinement [3]. This model is attractive because of its covariance and the transparent description of spontaneous chiral symmetry breaking. We construct the nucleon as a bound state solution of the relativistic Faddeev equation [4, 5, 6, 7] in the quark-diquark approximation [8], where both scalar and axial-vector diquark channels are included. This quark-diquark description of the single nucleon has the further advantage that it can be extended to finite baryon density [9]. We pay special attention to the polarized structure of the nucleon, comparing our results for the quark distributions with the empirical parameterizations.

\section{QUARK DISTRIBUTIONS}

The spin-dependent quark light-cone momentum distribution in the nucleon, at leading twist, is defined by Eq. (11), where $\psi_{q}$ is the quark field of flavour $q, x$ is the Bjorken scaling variable and the subscript $c$ reminds us

\footnotetext{
*Electronic address: icloet@physics.adelaide.edu.au

${ }^{\dagger}$ Electronic address: bentz@keyaki.cc.u-tokai.ac.jp

‡Electronic address: awthomas@jlab.org
}

that only connected matrix elements are included.

$$
\begin{aligned}
& \Delta f_{q}(x)=p_{-} \int \frac{d \xi^{-}}{2 \pi} e^{i x p^{+}} \xi^{-} \\
&\left\langle p, s\left|\bar{\psi}_{q}(0) \gamma^{+} \gamma_{5} \psi_{q}\left(\xi^{-}\right)\right| p, s\right\rangle_{c}
\end{aligned}
$$

We normalize the nucleon state vector according to noncovariant light-cone normalization: $\left\langle p, s\left|\bar{\psi}_{q} \gamma^{+} \psi_{q}\right| p, s\right\rangle_{c}=$ 3. The spin-independent distribution, $f_{q}(x)$, is defined by the replacement $\gamma^{+} \gamma_{5} \rightarrow \gamma^{+}$in Eq. (1). To determine the quark distributions in this model, it is convenient to express Eq. (1) in the form [10, 11]

$$
\begin{aligned}
\Delta f_{q}(x)=-i \int & \frac{d^{4} k}{(2 \pi)^{4}} \\
& \delta\left(x-\frac{k_{-}}{p_{-}}\right) \operatorname{Tr}\left(\gamma^{+} \gamma_{5} M_{q}(p, k)\right)
\end{aligned}
$$

where $M_{q}(p, k)$ is the quark two-point function in the bound nucleon. Hence, within any model that describes the nucleon as a bound state of quarks, the distribution functions can be associated with a straightforward Feynman diagram calculation.

The Feynman diagrams considered here are given in Fig. 1] where in our model the resulting distributions have no support for negative $x$. Therefore this is essentially a valence quark picture. By separating the isospin factors, the spin-dependent $u$ and $d$ distributions in the proton can be expressed as

$$
\begin{aligned}
\Delta u_{v}(x)=\Delta & f_{q / N}^{s}(x)+\frac{1}{2} \Delta f_{q(D) / N}^{s}(x)+\frac{1}{3} \Delta f_{q / N}^{a}(x) \\
& +\frac{5}{6} \Delta f_{q(D) / N}^{a}(x)+\frac{1}{2 \sqrt{3}} \Delta f_{q(D) / N}^{m}(x), \\
\Delta d_{v}(x)= & \frac{1}{2} \Delta f_{q(D) / N}^{s}(x)+\frac{2}{3} \Delta f_{q / N}^{a}(x) \\
& +\frac{1}{6} \Delta f_{q(D) / N}^{a}(x)-\frac{1}{2 \sqrt{3}} \Delta f_{q(D) / N}^{m}(x)
\end{aligned}
$$

The superscripts $s, a$ and $m$ refer to the scalar, axialvector or mixing terms, respectively, the subscript $q / N$ 


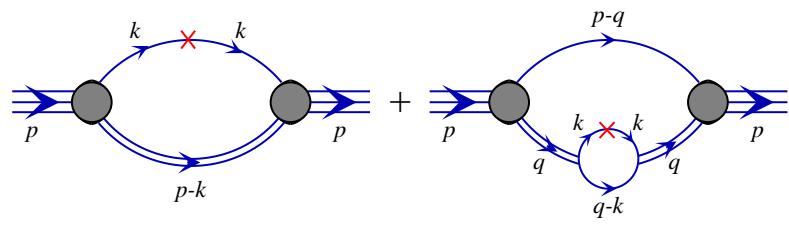

FIG. 1: Feynman diagrams representing the quark distributions in the nucleon, needed in the evaluation of Eq. (1). The single line represents the quark propagator and the double line the diquark $t$-matrix. The shaded oval denotes the quarkdiquark vertex function and the operator insertion has the form $\gamma^{+} \gamma_{5} \delta\left(x-\frac{k_{-}}{p_{-}}\right) \frac{1}{2}\left(1 \pm \tau_{z}\right)$ for the spin-dependent distribution and $\gamma^{+} \gamma_{5} \rightarrow \gamma^{+}$for the spin-independent case.

implies a quark diagram and similarly $q(D) / N$ a diquark diagram. Because the scalar diquark has spin zero, we have $\Delta f_{q(D) / N}^{s}(x)=0$ and hence the polarization of the $d$-quark arises exclusively from the axial-vector and the mixing terms. Similar expressions hold for the spinindependent distributions, but in that case there is no mixing contribution $\left(f_{q(D) / N}^{m}=0\right)[12$.

Importantly, in this covariant framework, the Ward identities corresponding to number and momentum conservation are satisfied, guaranteeing the validity of the baryon number and momentum sum rules [3, 13].

\section{THE NUCLEON IN THE NJL MODEL}

The NJL model is a chiral effective quark theory that is characterized by a 4 -Fermi contact interaction of the form, $\mathcal{L}_{I}=\sum_{i} G_{i}\left(\bar{\psi} \Gamma_{i} \psi\right)^{2}$, where the $\Gamma_{i}$ represent matrices in Dirac, colour and flavour space and $G_{i}$ are coupling constants 22]. Applying Fierz transformations, the interaction Lagrangian can be decomposed into various interacting $q \bar{q}$ and $q q$ channels. Writing only those terms relevant to this discussion, we have

$$
\mathcal{L}=\bar{\psi}(i \not \partial-m) \psi+\mathcal{L}_{I, \pi}+\mathcal{L}_{I, s}+\mathcal{L}_{I, a},
$$

where $m$ is the current quark mass. The interaction terms are given by

$$
\begin{aligned}
& \mathcal{L}_{I, \pi}=\frac{1}{2} G_{\pi}\left((\bar{\psi} \psi)^{2}-\left(\bar{\psi} \gamma_{5} \vec{\tau} \psi\right)^{2}\right), \\
& \mathcal{L}_{I, s}=G_{s}\left(\bar{\psi} \gamma_{5} C \tau_{2} \beta^{A} \bar{\psi}^{T}\right)\left(\psi^{T} C^{-1} \gamma_{5} \tau_{2} \beta^{A} \psi\right), \\
& \mathcal{L}_{I, a}=G_{a}\left(\bar{\psi} \gamma_{\mu} C \tau_{i} \tau_{2} \beta^{A} \bar{\psi}^{T}\right)\left(\psi^{T} C^{-1} \gamma^{\mu} \tau_{2} \tau_{i} \beta^{A} \psi\right),
\end{aligned}
$$

where $\beta^{A}=\sqrt{\frac{3}{2}} \lambda^{A}(A=2,5,7)$ are the colour $\overline{3}$ matrices and $C=i \gamma_{2} \gamma_{0}$. The familiar term $\mathcal{L}_{I, \pi}$ generates the constituent quark mass, $M$, via the gap equation and the pion as a $q \bar{q}$ bound state. The terms $\mathcal{L}_{I, s}$ and $\mathcal{L}_{I, a}$ represent the interactions in the scalar $\left(J^{\pi}=0^{+}, T=\right.$ 0$, colour $\overline{3})$ and axial-vector $\left(J^{\pi}=1^{+}, T=1\right.$, colour $\left.\overline{3}\right)$ diquark channels and are used to construct the nucleon as a quark-diquark bound state. The couplings $G_{\pi}, G_{s}$ and $G_{a}$ are related to the original couplings, $G_{i}$, via the Fierz transformation, but we use them here as free parameters which will be fixed by the properties of the pion and the nucleon.

Solving the appropriate Bethe-Salpeter equations, the standard NJL results for the diquark $t$-matrices are obtained [5, 12]. As explained in Ref. 8], these can be accurately approximated by the forms

$$
\begin{aligned}
\tau_{s}(q) & =4 i G_{s}-\frac{i g_{s}}{q^{2}-M_{s}^{2}}, \\
\tau_{a}^{\mu \nu}(q) & =4 i G_{a} g^{\mu \nu}-\frac{i g_{a}}{q^{2}-M_{a}^{2}}\left(g^{\mu \nu}-\frac{q^{\mu} q^{\nu}}{M_{a}^{2}}\right),
\end{aligned}
$$

which we also use here. The masses of the diquarks $M_{s}, M_{a}$ and their couplings to the quarks $g_{s}, g_{a}$ are defined as the poles and residues of the appropriate full diquark $t$-matrices.

The nucleon (quark-diquark) $t$-matrix satisfies the Faddeev equation

$$
T=Z+Z \Pi_{N} T=Z+T \Pi_{N} Z,
$$

where $Z$ is the quark exchange kernel and $\Pi_{N}$ the product of a quark propagator and a diquark $t$-matrix. In the non-covariant light-cone normalization used already in Eq. (11), the quark-diquark vertex function, $\Gamma_{N}$, is defined by the behaviour of $T$ near the pole

$$
T \stackrel{p_{+} \rightarrow \varepsilon_{p}}{\longrightarrow} \frac{\Gamma_{N} \bar{\Gamma}_{N}}{p_{+}-\varepsilon_{p}}
$$

where $\varepsilon_{p}=\frac{M_{N}^{2}}{2 p_{-}}$is the light-cone energy. Substituting this result into Eq. (11) gives the homogeneous Faddeev equations for the vertex functions

$$
\Gamma_{N}=Z \Pi_{N} \Gamma_{N}, \quad \text { and } \quad \bar{\Gamma}_{N}=\bar{\Gamma}_{N} \Pi_{N} Z .
$$

For this investigation we restrict ourselves to the static approximation, where we neglect the momentum dependence of the quark exchange kernel, $Z$. Including both scalar and axial-vector diquark channels, $Z$ takes the following form in the colour singlet and isospin- $\frac{1}{2}$ channel:

$$
Z=\frac{3}{M}\left(\begin{array}{cc}
1 & \sqrt{3} \gamma_{\mu^{\prime}} \gamma_{5} \\
\sqrt{3} \gamma_{5} \gamma^{\mu} & -\gamma_{\mu^{\prime}} \gamma^{\mu}
\end{array}\right)
$$

The quantity $\Pi_{N}$ effectively becomes the quark-diquark bubble graph:

$$
\Pi_{N}(p)=\int \frac{d^{4} k}{(2 \pi)^{4}} \tau(p-k) S(k),
$$

where

$$
\tau(q)=\left(\begin{array}{cc}
\tau_{s}(q) & 0 \\
0 & \tau_{a}^{\mu \nu}(q)
\end{array}\right) .
$$


The eigenfunction of the kernel $K \equiv Z \Pi_{N}$, in Eq. (13), has the following form, up to normalization:

$$
\Gamma(p, s)=\left[\begin{array}{c}
\alpha_{1} \\
\alpha_{2} \frac{p^{\mu}}{M_{N}} \gamma_{5}+\alpha_{3} \gamma^{\mu} \gamma_{5}
\end{array}\right] u_{N}(p, s),
$$

where the upper and lower component refer to the scalar and axial-vector diquark channels, respectively and $u_{N}$ is a free Dirac spinor with mass $M_{N}$. We choose the normalization $\bar{u}_{N} u_{N}=1=\bar{\Gamma} \Gamma{ }^{1}$ Inserting this form into Eq. (13) gives three homogeneous equations for the $\alpha$ 's and the nucleon mass $M_{N}$ is determined by the requirement that the eigenvalue of $K$, in Eq. (13), equal 1.

The normalization of the vertex function follows from the definition given in Eq. (12), we obtain

$$
\Gamma_{N}(p, s)=\sqrt{-Z_{N} \frac{M_{N}}{p_{-}}} \Gamma(p, s),
$$

where

$$
Z_{N}=\frac{p_{-}}{M_{N}} \frac{-1}{\Gamma(p) \frac{\partial \Pi_{N}(p)}{\partial p_{+}} \Gamma(p)}
$$

As with any non-renormalizable theory a regularization prescription must be specified to fully define the model. We choose the proper-time regularization scheme [8, 14, 15, 16], where loop integrals of products of propagators are evaluated by introducing Feynman parameters, Wick rotating and making the denominator replacement

$$
\frac{1}{X^{n}} \longrightarrow \frac{1}{(n-1) !} \int_{1 /\left(\Lambda_{U V}\right)^{2}}^{1 /\left(\Lambda_{I R}\right)^{2}} d \tau \tau^{n-1} e^{-\tau X}
$$

where $\Lambda_{I R}$ and $\Lambda_{U V}$ are, respectively, ultraviolet and infrared cutoffs. The former has the effect of eliminating unphysical thresholds for hadron decay into quarks, hence simulating an important aspect of confinement 15 .

\section{RESULTS}

The parameters of the model are $\Lambda_{I R}, \Lambda_{U V}, m, G_{\pi}$, $G_{s}$ and $G_{a}$. The infrared scale is expected to be of or$\operatorname{der} \Lambda_{Q C D}$ and we set it to $\Lambda_{I R}=0.28 \mathrm{GeV}$. This is slightly larger than our previous work [3], because our studies of the saturation properties of nuclear matter favour this [9]. The parameters $m, \Lambda_{U V}$ and $G_{\pi}$ are determined by requiring $M=400 \mathrm{MeV}$ via the gap equation, $f_{\pi}=93 \mathrm{MeV}$ from the familiar one loop pion decay

\footnotetext{
1 The conjugate vertex function, $\bar{\Gamma}$, which is a left eigenfunction of $\bar{K} \equiv \Pi_{N} Z$, is obtained by taking the ordinary hermitian conjugate of $\Gamma$ and introducing a minus sign for the axial-vector components.
}

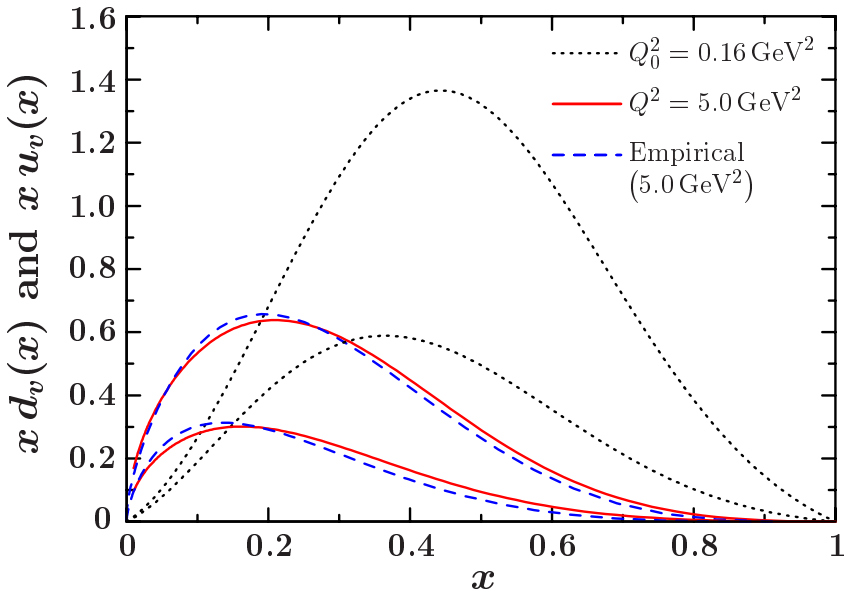

FIG. 2: Spin-independent valence $u$ and $d$ distributions multiplied by Bjorken $x$. There are three curves for each quark flavour, with the lower curve of each type representing the $d$ distribution. The dotted line is the model prediction at the NJL scale of $Q_{0}^{2}=0.16 \mathrm{GeV}^{2}$ and the solid line is the result after QCD evolution to the scale $Q^{2}=5.0 \mathrm{GeV}^{2}$. The dashed line is the empirical parametrization of Ref. [18], at the scale $Q^{2}=5.0 \mathrm{GeV}^{2}$.

diagram and $m_{\pi}=140 \mathrm{MeV}$ from the pole of the $q \bar{q} t$ matrix in the pion channel. This gives $m=15.3 \mathrm{MeV}$, $\Lambda_{U V}=0.66 \mathrm{GeV}$ and $G_{\pi}=17.81 \mathrm{GeV}^{-2}$. The couplings $G_{s}$ and $G_{a}$ are determined by reproducing the nucleon mass $M_{N}=940 \mathrm{MeV}$ as the solution of Eq. (13) and satisfying the Bjorken sum rule within our model, where $g_{A}=1.267$. We obtain $G_{s}=8.41 \mathrm{GeV}^{-2}$ and $G_{a}=1.36 \mathrm{GeV}^{-2}$. With these model parameters the diquark masses are $M_{s}=0.65 \mathrm{GeV}$ and $M_{a}=1.2 \mathrm{GeV}$ and the coefficients in the nucleon vertex function, Eq. (17), are $\left(\alpha_{1}, \alpha_{2}, \alpha_{3}\right)=(-0.35,-0.0088,0.47)$.

To compare the predictions of the model with experimental data as well as the empirical parameterizations, it is necessary to determine the model scale, $Q_{0}^{2}$. We do this by optimizing $Q_{0}^{2}$ such that the spin-independent distribution, $u_{v}(x)$, best reproduces the empirical parameterization after $Q^{2}$ evolution. We find a model scale of $Q_{0}^{2}=0.16 \mathrm{GeV}^{2}$, which is typical of valence dominated models [12, 13, 17].

Results for the spin-independent and spin-dependent valence $u$ and $d$ distributions are presented in Figs. 2 and 31 respectively. We show the predictions at the model scale and after QCD evolution ${ }^{2}$ to $Q^{2}=5 \mathrm{GeV}^{2}$, where they are compared to empirical parameterizations. We find excellent agreement between the model results and the parameterizations. In comparison with the pure

\footnotetext{
${ }^{2}$ We utilize the computer program of Ref. [20] for the spinindependent case and of Ref. [21] for the spin-dependent case. We choose DGLAP evolution with $N_{f}=3, \Lambda_{\mathrm{QCD}}=250 \mathrm{MeV}$ in the $\overline{\mathrm{MS}}$ renormalization scheme up to NLO.
} 


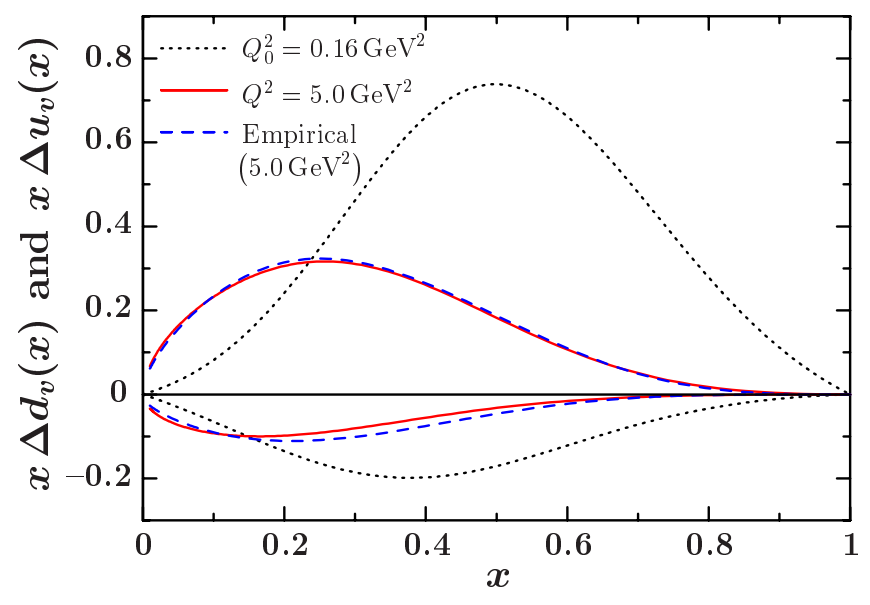

FIG. 3: Spin-dependent valence $u$ and $d$ distributions multiplied by Bjorken $x$. The curves are as in Fig. 2 with empirical parameterizations taken from Ref. [19].

scalar model [3, 22], the agreement has improved substantially, especially for the spin-dependent case.

Our model results for the first polarized moments are $\Delta u_{v}=0.924$ and $\Delta d_{v}=-0.343$ which agree with the values $\Delta u_{v}=0.926 \pm 0.014$ and $\Delta d_{v}=-0.341 \pm 0.018$ determined from the axial coupling constants of octet baryons discussed in Ref. [23]. This emphasizes the importance of including axial-vector diquark correlations, since the pure scalar model would give a vanishing $\Delta d_{v}$ and a somewhat smaller $\Delta u_{v}$. The spin sum in our model is $\Delta \S=0.581$, which is smaller than the result of the pure scalar model, but still somewhat larger than the accepted value of $\Delta \S=0.213 \pm 0.138[19]$. This discrepancy primarily reflects the absence of the $U(1)$ axial anomaly [24, 25].

The behaviour of structure function and hence quark distribution ratios at large $x$ has been an area of considerable debate [26, 27] and is one of the regions where perturbative QCD (pQCD) offers firm predictions [28]. Experimentally, the ratio $d(x) / u(x)$ is surprisingly poorly known [29]. In the limit $x \rightarrow 1$ it is thought to lie somewhere between 0 , the prediction based on scalar diquark dominance [30] and $\frac{1}{5}$, the pQCD result [28]. Analysis in Ref. 26] favours the pQCD prediction. The same predictions also hold for the spin-dependent ratio, $\Delta d(x) / \Delta u(x)$, as $x$ approaches 1 .

In Fig. 4 we plot our results for the ratios $d_{v}(x) / u_{v}(x)$ and $\Delta d_{v}(x) / \Delta u_{v}(x)$, together with the ratios of the empirical distributions. The $x \rightarrow 1$ limit of the spinindependent ratio is in agreement with the pQCD result. The spin-dependent ratio, however, approaches $\sim-\frac{1}{16}$, the opposite sign to the pQCD prediction. Although the empirical parameterizations are constrained to give 0 for these ratios as $x \rightarrow 1$, we note that the systematic errors in both empirical ratios are very large in the region $x \gtrsim 0.5$ 19, 32, 33, 34].

It is important to note that the pQCD predictions for the mixed flavour ratios are somewhat model depen-

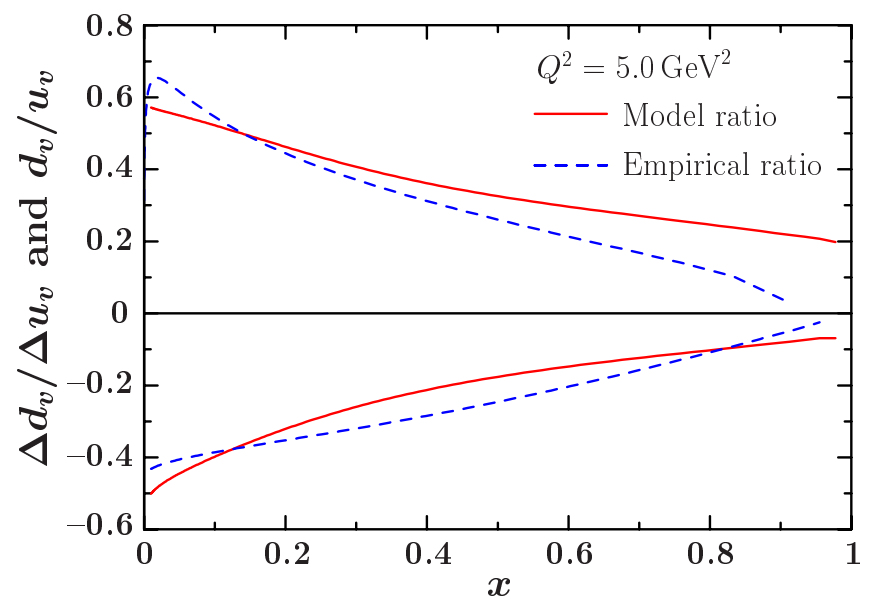

FIG. 4: Mixed flavour ratios for spin-independent and spindependent distributions. There are two curves for each ratio, with the lower curves the polarized result. The curves are as in Fig. 2 with spin-independent parameterizations taken from Ref. [18] and the spin-dependent from Ref. [19]

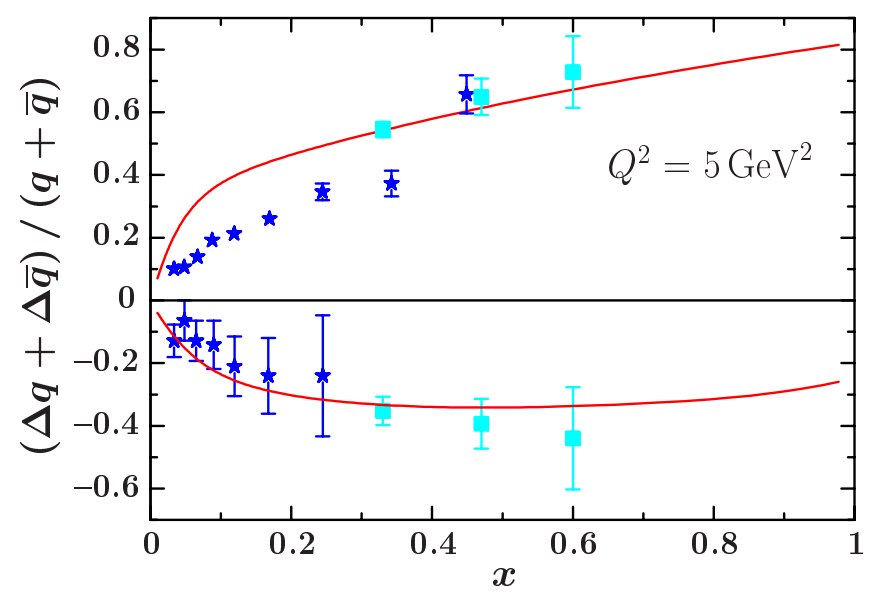

FIG. 5: Single flavour ratios $(\Delta q+\Delta \bar{q}) /(q+\bar{q})$ where $q \in$ $(u, d)$, at the scale $Q^{2}=5.0 \mathrm{GeV}^{2}$. The lower curve represents the $d$-quark distribution. The experimental results are from Hall A at Jefferson Lab 27] (solid squares) and Hermes [31] (solid stars).

dent, as assumptions have to be made about the relative strengths of the $u$ and $d$ contributions to the nucleon wavefunction. A more rigorous $\mathrm{pQCD}$ prediction, relying only on helicity conservation, is possible for the single flavour ratios $\Delta u(x) / u(x)$ and $\Delta d(x) / d(x)$. Perturbative QCD predicts that both these ratios should approach 1 for large $x$, which would require a change of sign in the $\Delta d$ distribution.

In Fig. 5 we plot our results for the ratios $(\Delta q+\Delta \bar{q}) /(q+\bar{q})$ where $q \in(u, d)$. Since we wish to compare these ratios directly to recent experimental data, we include sea quark distributions generated through the $Q^{2}$ evolution. In the $x \rightarrow 1$ limit our model ratios approach $\approx 0.8$ for the $u$-quark and $\approx-0.25$ for the 


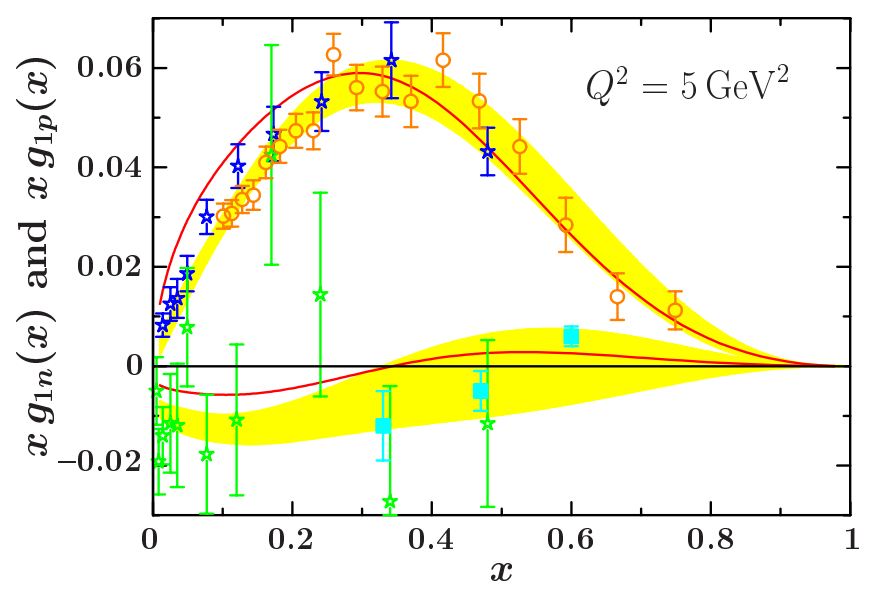

FIG. 6: Polarized structure functions $g_{1 p}$ and $g_{1 n}$ at $Q^{2}=$ $5 \mathrm{GeV}^{2}$. The solid line is the model prediction, with the lower curve corresponding to $g_{1 n}$. The shaded areas represent the empirical parameterizations with uncertainties of Ref. 32], at the same scale. The experimental data, with $1 \leq Q^{2} \leq 10 \mathrm{GeV}^{2}$, is from SMC 36] (open stars), SLAC E143 [38] (open circles) and JLab 27] (solid squares).

$d$-quark. This seeming contradiction to pQCD has also been suggested by recent experiments by the Jefferson Lab Hall A collaboration 27, 35], with our predictions consistent with their experimental results. This data is also shown in Fig. [5

In Fig. 6] we give our results for the spin-dependent structure functions $g_{1 p}(x)$ and $g_{1 n}(x)$. The parameterizations of Ref. 32] are also included as the shaded areas, which indicate the empirical uncertainties. Our results compare well with the empirical parameterizations, agreeing within uncertainties for the region $x \gtrsim 0.25$. Comparison with experiment is also favorable, although the experimental determination for $g_{1 n}(x)$ is less certain.

Model results for the asymmetries $A_{1 p}(x)$ and $A_{1 n}(x)$ are given in Fig. 7 where we see good agreement in the valence region. However, for small $x, A_{1 p}(x)$ is slightly too large, because of the enhancement of $g_{1 p}(x)$ in the same region. This is most likely associated with the omission of the effects of the axial anomaly in the present work. It is also clear from the experimental data that the uncertainties in these ratios at large $x$, are still significant.

\section{CONCLUSION}

Using a covariant quark-diquark model for the nucleon, including both scalar and axial-vector diquark channels, we calculated the spin-independent and spin-dependent quark light-cone momentum distributions and structure functions. A key feature of the framework is that it produces quark distributions that have the correct support and obey the number and momentum sum rules. The model also incorporates important aspects of confinement by eliminating unphysical thresholds for nucleon

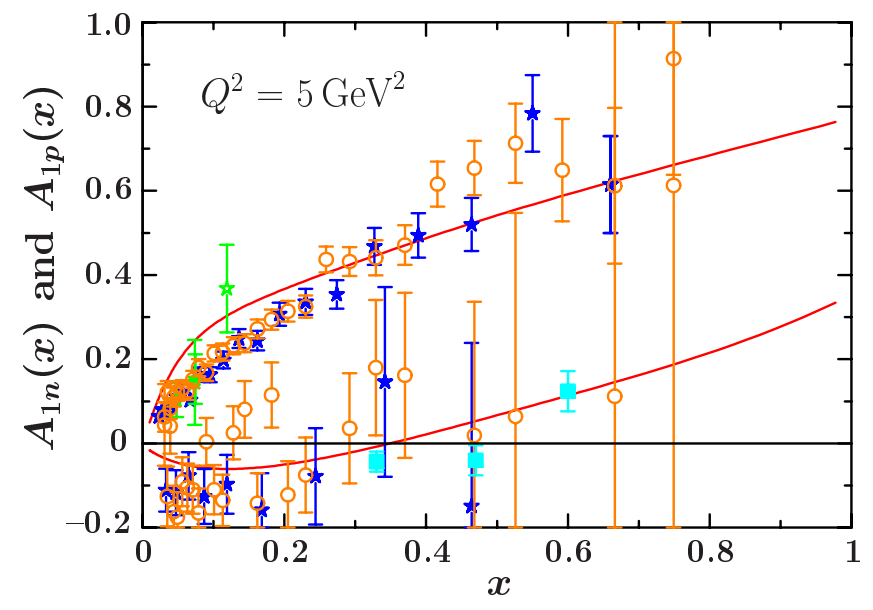

FIG. 7: Structure function ratios $A_{1 p}$ and $A_{1 n}$, at the scale $Q^{2}=5, \mathrm{GeV}^{2}$. The solid line is the model prediction, with the lower line corresponding to $A_{1 n}$. The experimental points represent data with $1 \leq Q^{2} \leq 10 \mathrm{GeV}^{2}$ from Hermes 37] (closed star), SMC [36] (open star), E143 [38] (open circles) and JLab 27] (solid squares). We do not plot the E155 [39] results as they are similar to those from E143.

decay into quarks.

Highlights of our results are obtaining values for the polarized first moments of the quark distributions $\Delta u_{v}=$ 0.924 and $\Delta d_{v}=-0.343$, in agreement with those obtained from axial couplings of octet baryons. We also obtain excellent agreement with empirical parameterizations of the valence quark distributions. We paid special attention to the single flavour ratios $(\Delta q+\Delta \bar{q}) /(q+\bar{q})$ and the asymmetries $A_{1 p}$ and $A_{1 n}$, finding good agreement with recent experimental results from JLab.

These results indicate that diquark correlations are an essential feature of the non-perturbative structure of the nucleon. In particular, the admixture of axial-vector diquarks, though small, is essential to obtain the observed agreement with empirical data.

Finally, we would like to mention that a very important advantage of this covariant quark-diquark model is that it can be readily extended to the case of finite nucleon density. The results presented in this paper strongly suggest that this model should provide a reliable basis from which to begin investigation of the medium modifications of both spin-independent and spin-dependent structure functions.

\section{Acknowledgments}

IC and WB thank W. Melnitchouk and H. Mineo for interesting and helpful discussions. This work was supported by the Australian Research Council and DOE contract DE-AC05-84ER40150, under which SURA operates Jefferson Lab, and by the Grant in Aid for Scientific Research of the Japanese Ministry of Education, Culture, Sports, Science and Technology, Project No. C216540267. 
[1] J. Ashman et al. [European Muon Collaboration], Phys. Lett. B 206, 364 (1988).

[2] Y. Nambu and G. Jona-Lasinio, Phys. Rev. 122, 345 (1961).

[3] H. Mineo, W. Bentz, N. Ishii, A. W. Thomas and K. Yazaki, Nucl. Phys. A 735, 482 (2004) arXiv:nucl-th/0312097.

[4] S. z. Huang and J. Tjon, Phys. Rev. C 49, 1702 (1994) arXiv:hep-ph/9308362.

[5] N. Ishii, W. Bentz and K. Yazaki, Nucl. Phys. A 587, 617 (1995).

[6] M. Oettel, R. Alkofer and L. von Smekal, Eur. Phys. J. A 8, 553 (2000) arXiv:nucl-th/0006082.

[7] P. Maris and C. D. Roberts, Int. J. Mod. Phys. E 12, 297 (2003) arXiv:nucl-th/0301049.

[8] W. Bentz and A. W. Thomas, Nucl. Phys. A 696, 138 (2001).

[9] I. C. Cloët, W. Bentz and A. W. Thomas, arXiv:nucl-th/0504019

[10] R. L. Jaffe, MIT-CTP-1261 Lectures presented at the Los Alamos School on Quark Nuclear Physics, Los Alamos, N.Mex., Jun 10-14, 1985.

[11] V. Barone, A. Drago and P. G. Ratcliffe, Phys. Rept. 359, 1 (2002).

[12] H. Mineo, W. Bentz, N. Ishii and K. Yazaki, Nucl. Phys. A 703, 785 (2002) arXiv:nucl-th/0201082.

[13] H. Mineo, W. Bentz and K. Yazaki, Phys. Rev. C 60, 065201 (1999) arXiv:nucl-th/9907043.

[14] D. Ebert, T. Feldmann and H. Reinhardt, Phys. Lett. B 388, 154 (1996).

[15] G. Hellstern, R. Alkofer and H. Reinhardt, Nucl. Phys. A 625, 697 (1997).

[16] J. S. Schwinger, Phys. Rev. 82, 664 (1951).

[17] A. W. Schreiber, A. I. Signal and A. W. Thomas, Phys. Rev. D 44, 2653 (1991).

[18] A. D. Martin, R. G. Roberts, W. J. Stirling and R. S. Thorne, Phys. Lett. B 531, 216 (2002) arXiv:hep-ph/0201127.

[19] M. Hirai, S. Kumano and N. Saito, Phys. Rev. D 69, 054021 (2004).

[20] M. Miyama and S. Kumano, Comput. Phys. Commun. 94, 185 (1996) arXiv:hep-ph/9508246.
[21] M. Hirai, S. Kumano and M. Miyama, Comput. Phys. Commun. 108, 38 (1998).

[22] I. C. Cloët, W. Bentz and A. W. Thomas, Nucl. Phys. B Proc. Suppl. 141, 225 (2005).

[23] Y. Goto et al. [Asymmetry Analysis collaboration], Phys. Rev. D 62, 034017 (2000) arXiv:hep-ph/0001046.

[24] G. Altarelli and G. G. Ross, Phys. Lett. B 212, 391 (1988).

[25] A. V. Efremov and O. V. Teryaev, JINR-E2-88-287.

[26] W. Melnitchouk and A. W. Thomas, Phys. Lett. B 377, 11 (1996) arXiv:nucl-th/9602038.

[27] X. Zheng et al. [Jefferson Lab Hall A Collaboration], Phys. Rev. C 70, 065207 (2004) arXiv:nucl-ex/0405006.

[28] G. R. Farrar and D. R. Jackson, Phys. Rev. Lett. 35, 1416 (1975); S. J. Brodsky, M. Burkardt and I. Schmidt, Nucl. Phys. B 441, 197 (1995) arXiv:hep-ph/9401328.

[29] M. Botje, Eur. Phys. J. C 14, 285 (2000) arXiv:hep-ph/9912439.

[30] F. E. Close, Phys. Lett. B 43, 422 (1973); F. E. Close and A. W. Thomas, Phys. Lett. B 212, 227 (1988).

[31] K. Ackerstaff et al. [HERMES Collaboration], Phys. Lett. B 464, 123 (1999) arXiv:hep-ex/9906035.

[32] J. Blumlein and H. Bottcher, Nucl. Phys. B 636, 225 (2002) arXiv:hep-ph/0203155.

[33] A. D. Martin, R. G. Roberts, W. J. Stirling and R. S. Thorne, Eur. Phys. J. C 28, 455 (2003) arXiv:hep-ph/0211080.

[34] A. D. Martin, R. G. Roberts, W. J. Stirling and R. S. Thorne, Eur. Phys. J. C 35, 325 (2004) arXiv:hep-ph/0308087.

[35] X. Zheng et al. [Jefferson Lab Hall A Collaboration], Phys. Rev. Lett. 92, 012004 (2004) arXiv:nucl-ex/0308011.

[36] B. Adeva et al. [Spin Muon Collaboration], Phys. Rev. D 58, 112002 (1998).

[37] A. Airapetian et al. [HERMES Collaboration], Phys. Lett. B 442, 484 (1998) arXiv:hep-ex/9807015.

[38] K. Abe et al. [E143 collaboration], Phys. Rev. D 58, 112003 (1998) arXiv:hep-ph/9802357.

[39] P. L. Anthony et al. [E155 Collaboration], Phys. Lett. B 493, 19 (2000) arXiv:hep-ph/0007248. 